\title{
A phase II study of the histone deacetylase inhibitor vorinostat combined with tamoxifen for the treatment of patients with hormone therapy-resistant breast cancer
}

\author{
PN Munster*,', KT Thurn', S Thomas', P Raha', M Lacevic², A Miller', M Melisko', R Ismail-Khan², H Rugo', \\ M Moasser' and SE Minton ${ }^{2}$
}

'Division of Hematology and Oncology, University of California, San Francisco, 1600 Divisadero, Rm A719 Box 17II, San Francisco, CA 94I43, USA;

${ }^{2} \mathrm{H}$. Lee Moffitt Cancer Center and Research Institute, 12902 Magnolia Drive Tampa, FL 336 I2, USA

BACKGROUND: Histone deacetylases (HDACs) are crucial components of the oestrogen receptor (ER) transcriptional complex. Preclinically, HDAC inhibitors can reverse tamoxifen/aromatase inhibitor resistance in hormone receptor-positive breast cancer. This concept was examined in a phase II combination trial with correlative end points.

METHODS: Patients with ER-positive metastatic breast cancer progressing on endocrine therapy were treated with $400 \mathrm{mg}$ of vorinostat daily for 3 of 4 weeks and $20 \mathrm{mg}$ tamoxifen daily, continuously. Histone acetylation and HDAC2 expression in peripheral blood mononuclear cells were also evaluated.

RESULTS: In all, 43 patients (median age 56 years (3I-7I)) were treated, 25 (58\%) received prior adjuvant tamoxifen, 29 (67\%) failed one prior chemotherapy regimen, 42 (98\%) progressed after one, and 23 (54\%) after two aromatase inhibitors. The objective response rate by Response Evaluation Criteria in Solid Tumours criteria was 19\% and the clinical benefit rate (response or stable disease $>24$ weeks) was 40\%. The median response duration was 10.3 months (confidence interval: 8.1-12.4). Histone hyperacetylation and higher baseline HDAC2 levels correlated with response.

CONCLUSION: The combination of vorinostat and tamoxifen is well tolerated and exhibits encouraging activity in reversing hormone resistance. Correlative studies suggest that HDAC2 expression is a predictive marker and histone hyperacetylation is a useful pharmacodynamic marker for the efficacy of this combination.

British Journal of Cancer (201 I) I 04, I828-1835. doi:I0.1038/bjc.201 I.I56 www.bjcancer.com

Published online 10 May 20II

(C) 201 I Cancer Research UK

Keywords: histone deacetylase; HDAC; HDAC inhibitors; breast cancer; oestrogen receptor; anti-oestrogen therapy

Despite a decrease in incidence, an expected 194280 women will present with breast cancer in the United States in 2010, resulting in over 40000 deaths (Jemal et al, 2010). In more than two thirds of these women, tumours express either oestrogen receptors (ERs) or progesterone receptors (PgRs), which are frequently less sensitive to chemotherapy (EBCTCG, 2005), but are amenable to hormonal therapy. The most commonly used strategies for pharmacological inhibition of ER signalling are treatment with anti-oestrogens or aromatase inhibitors. For patients with metastatic disease, the response rate to first-line hormonal therapy with anti-oestrogens or aromatase inhibitors ranges from $21 \%$ to $33 \%$ (Nabholtz et al, 2000; Bonneterre et al, 2001; Mouridsen et al, 2001; Chia et al, 2008). The objective response rates of second-line hormonal therapies, such as exemestane or fulvestrant, measured by Response Evaluation Criteria in Solid Tumours (RECIST) criteria in a recent trial were $6.7 \%$ and $7.4 \%$, respectively (Chia et al, 2008). A study of low dose estradiol as second- or third-line therapy

\footnotetext{
*Correspondence: Professor PN Munster;

E-mail: pmunster@medicine.ucsf.edu

Received 20 December 2010; revised 23 February 2011; accepted 7

April 20 I ; published online I0 May 201 I
}

showed stable disease, but no objective responses (Ellis et al, 2009). Thus, novel approaches to reverse hormone therapy resistance are needed.

Histone deacetylases (HDACs) and histone acetyltransferases have important roles in the maintenance and function of chromatin by regulating the acetylation of histones. Recent data suggest that HDACs and histone acetyltransferases regulate the acetylation of many non-histone targets and therefore may represent a key means of post-translational regulation beyond their established roles in transcriptional regulation. Biologically, HDAC inhibitors induce growth arrest, differentiation, and cell death in breast cancer cells. Despite clinical efficacy in patients with cutaneous T-cell lymphomas, the therapeutic window of the currently available HDAC inhibitors may not suffice for meaningful anti-tumour efficacy in breast cancer when used as a single agent without more careful patient selection or the definition of a biomarker (Luu et al, 2008).

In preclinical models, treatment of ER-positive breast cancer cells with HDAC inhibitors leads to transcriptional downregulation and protein modification of the ER (Yi et al, 2008). Treatment with an HDAC inhibitor reverses tamoxifen-induced ER stabilisation, which is followed by induction of pro-apoptotic genes and apoptotic cell death (Hodges-Gallagher et al, 2006; Bicaku et al, 
2008; Thomas et al, 2011). Potentiation of tamoxifen by the HDAC inhibitor vorinostat has been shown in preclinical models at clinically achievable and tolerable concentrations (Kelly et al, 2005; Hodges-Gallagher et al, 2006; Galanis et al, 2009; Munster et al, $2009 \mathrm{~b})$. Epigenetic modulation of ER signalling by HDAC inhibitors may therefore represents a novel strategy to reverse hormone therapy resistance in advanced breast cancer. Further studies suggest that HDAC1 and 2 may have an important role in the regulation of oestrogen signalling and may therefore be relevant targets for HDAC inhibitor activity.

Prior studies with HDAC inhibitors suggest that these agents have a relatively short terminal half-life, with that of vorinostat reported to range from 21 to $58 \mathrm{~min}$ (Kelly et al, 2003). However, the pharmacodynamic effects often exceed the plasma half-life of these drugs, suggesting that pharmacodynamic measures may be a better predictor of tissue drug exposure than pharmacological values. Furthermore, several studies suggest vorinostat levels vary considerably between patients (Kelly et al, 2003; O'Connor et al, 2006). This may account for the poor correlation between vorinostat plasma levels and change in histone acetylation, a biomarker for molecular response (Munster et al, 2009b). A pharmacodynamic assay has therefore been developed by our laboratory to measure histone acetylation and HDAC enzyme expression in peripheral blood mononuclear cells (PBMCs) to allow for a more reliable means to measure the target activity of HDAC inhibitors in this study. Published data from a previous trial suggest that the change in histone acetylation in in vitro models, PBMCs, and tumour cells is comparable (Hodges-Gallagher et al, 2006; Bicaku et al, 2008).

Therefore, the objectives of this phase II trial were (1) to evaluate the toxicity of vorinostat and tamoxifen when administered in patients with hormone receptor-positive breast cancer after progression on prior hormone therapy, (2) to estimate the anti-tumour activity of vorinostat and tamoxifen in this patient population, and (3) to characterise the pharmacodynamic profile of histone acetylation and HDAC2 expression.

\section{MATERIALS AND METHODS}

\section{Patient selection}

Pre- and post-menopausal women with ER- or PgR-positive metastatic breast cancer with (1) progression on any number of aromatase inhibitors for metastatic disease or (2) recurrence of disease while on adjuvant aromatase inhibitors or (3) premenopausal women who had completed tamoxifen for at least 1 year were eligible for this trial who did not wish to undergo ovarian suppression in conjunction with aromatase inhibitor treatment. Prior treatment with tamoxifen or fulvestrant was permitted in the adjuvant setting, yet not for metastatic disease. Patients were allowed to have up to three prior chemotherapy regimens for metastatic disease. Bone-only disease was permissible if at least one lesion measured $1 \mathrm{~cm}$ by magnetic resonance imaging. Measurable disease, as defined by RECIST 1.0, was required. Other eligibility criteria were (1) Eastern Cooperative Group performance status of 0 , 1, or 2; (2) adequate bone marrow function (white blood cell count $\geqslant 3000$ per $\mathrm{mm}^{3}$, absolute neutrophil count $\geqslant 1500$ per $\mathrm{mm}^{3}$, and platelets $\geqslant 100000$ per $\mathrm{mm}^{3}$ ), adequate hepatic function (aspartate aminotransferase, alanine aminotransferase, and alkaline phosphatase levels $\leqslant 2.5$ times upper limit of institutional normal, total bilirubin $\leqslant 2.0 \mathrm{mg} \mathrm{dl}^{-1}$ ), and adequate renal function (serum creatinine $\leqslant 1.8 \mathrm{mg} \mathrm{dl}^{-1}$ or creatinine clearance $>60 \mathrm{ml}$ per minute times upper limit of institutional normal); (3) no prior treatment with HDAC inhibitors or other therapies for breast cancer within the preceding 3 weeks, (4) no prior radiation to the only measurable lesion; (5) no active intercurrent medical condition; (6) no vaginal bleeding, known endometrial hyperplasia, or cancer; and (7) no other invasive malignancies within the last 5 years, with the exception of non-melanoma skin cancer and in situ cervical cancer, (8) patients with brain metastases had to demonstrate stability for at least 90 days. Patients with prior thromboembolic events were to be on therapeutic anti-coagulation during the entire time of study.

All participants provided written informed consent, and participating institutions obtained annual Institutional Review Board approval in accordance with federal, state, local, and institutional requirements and guidelines.

\section{Drug administration, safety, and response assessment}

Patients received $400 \mathrm{mg}$ of vorinostat once daily for 21 of 28 days and $20 \mathrm{mg}$ tamoxifen daily without interruption. Both drugs were given in an oral formulation. A cycle consisted of 28 days. Patients were allowed to remain on treatment until disease progression or the emergence of unacceptable toxicity. Toxicity assessment, interim history and physical exams, complete blood count, and serum chemistry profile chemistries (electrolytes, blood urea nitrogen, creatinine, magnesium, calcium, phosphate, aspartate aminotransferase, alkaline phosphatase, and total bilirubin) were obtained at baseline, weekly in cycle one, and then on day 1 of every cycle.

Tests for tumour markers and documentation of measurable disease by computed tomography (CT) to evaluate response were performed after every two cycles. Adverse events and other symptoms were graded according to the NCI Common Terminology Criteria of Adverse Events (CTCAE) version 3.0. Both agents were given at the full prescribing dose. As this was the first trial to combine vorinostat with tamoxifen, we performed additional safety evaluations during the enrolment of the first 15 patients with the mandate to halt the trial in the case where grade 3 and 4 toxicities were seen in 4 or more of the first 15 patients. A reduction to $75 \%$ of the vorinostat dose was required for patients experiencing grade 3 non-haematological toxicity and grade 4 haematological toxicity after recovery of toxicities with a second dose modification to $50 \%$. If toxicities persisted, patients were withdrawn from the study. Consistent with standard practice, the tamoxifen dose was reduced for tamoxifen-specific toxicity only.

\section{Pharmacodynamics: histone acetylation and HDAC expression}

Peripheral blood mononuclear cells obtained pre-treatment and day 8 post-treatment in cycle 1 were isolated by Ficoll centrifugation (Ficoll-Paque, GE HealthCare, Piscataway, NJ, USA). Cells were spun onto glass slides, fixed in $5 \%$ acetic acid/95\% ethyl alcohol, and blocked with $2 \%$ bovine serum albumin. Histone $\mathrm{H} 4$ acetylation (rabbit polyclonal, Upstate Biotechnology, Lake Placid, NY, USA), and HDAC2 (monoclonal, Upstate Biotechnology) were visualised using fluorescently labelled secondary antibodies. Slides were counter stained with an antibody control directed against pan-histone H3 (monoclonal, BD Biosciences, Franklin Lakes, NJ, USA) for acetyl-H4 and polyclonal, Upstate Biotechnology for HDAC2) and evaluated by immunofluorescence microscopy (Zeiss Axio Imager.Z2, Carl Zeiss Inc., Thornwood, NY, USA). Resultant images were analysed as previously described (Munster et al, 2007, 2009a). Briefly, the mean pixel intensity for each epitope was determined within the nucleus from acquired images using Adobe Photoshop software. The mean pixel intensity for each epitope was determined adjacent to each cell and subtracted from the nuclear value to control for background staining. Pre- and post-treatment histone acetylation and HDAC2 expression were normalised to panhistone $\mathrm{H} 3$ expression (Munster et al, 2007, 2009a). At least 100 cells were evaluated from two slides for each time point and condition. Changes in $\mathrm{H} 4$ histone acetylation were further verified by western blot when sufficient sample was available (Munster et al, 2007, 2009a). In brief, total cell extracts $(10 \mu \mathrm{g})$ were separated by SDSPAGE and probed with primary antibodies against histone acetyl-H4 
and pan-histone H3 (polyclonal, Upstate Biotechnology). Protein levels were quantified by densitometry using Image J software (NIH).

\section{Statistical design and methods}

The study employed a Simon two-stage design with early stopping rules to preempt enrolment in the event of insufficient activity or excessive toxicity (Chen and $\mathrm{Ng}, 1998$ ). During the first stage, 18 patients were to be enrolled and evaluated. If at least two responses were observed among the first 18 patients, an additional 25 patients were to be enrolled in a second phase. The regimen would be considered inactive if seven or fewer patients responded. If the 'true' response probability is $10 \%$, the average probability to end the trial early would be $73.38 \%$. Conversely, if the 'true' response probability is $25 \%$, there is a $13.53 \%$ probability to incorrectly classify the trial as inactive. Pearson's correlation coefficient methods were used to estimate correlations between two variables and to perform the test of significance of the estimated correlation with two-sided $P$-values at a 0.05 significance level (SAS version 9.1, SAS Institute Inc., Cary, NC, USA). Early stopping rules were set for dose-limiting toxicities to occur in no more than $33 \%$ of patients in the first cycle.

\section{RESULTS}

\section{Patient characteristics}

The study (clinicaltrials.gov identifier number NCT00365599) enrolled 43 evaluable patients at the Moffitt Cancer Center in Tampa, Florida and the University of California, San Francisco. One patient withdrew consent after 2 weeks and had to be replaced as pre-specified by the protocol. Patient characteristics and demographics are listed in Table 1.

\section{Adverse events}

A summary of adverse events by CTCAE (version 3.0) for all the patients is listed in Table 2. As in standard medical practice, dose reduction for tamoxifen was not performed for any toxicities, but tamoxifen was stopped in one patient after she developed a thromboembolic event. Vorinostat was given 21 of 28 days of each cycle, whereas tamoxifen was given continuously. This schedule allowed for the distinction between vorinostat toxicities and toxicities associated with the combination. The predominant toxicities of vorinostat included fatigue and anorexia, neutropenia, lymphopenia, and thrombocytopenia. Dose adjustments were made for all patients with drug-related grade 3 and grade 4 toxicities associated with vorinostat. Dose adjustments to $300 \mathrm{mg}$ vorinostat for grade 3 toxicities in 13 patients (30\%) and to $200 \mathrm{mg}$ for grade 4 toxicities or persistent grade 3 toxicities in 7 patients $(16 \%)$ were required. These included four (9\%) patients requiring dose reductions for neutropenia as the initial event, four $(9 \%)$ for thrombocytopenia, seven (16\%) for fatigue, and one each $(2 \%)$ for weight loss, liver enzyme elevation, and diarrhoea. Three patients experienced pulmonary emboli, two of which were incidental findings on CT scans. One patient had a symptomatic pulmonary embolus after a long transcontinental flight. In 13 of the 20 patients, dose reductions were required after the first cycle. Alopecia occurred in four patients after prolonged exposure (cycle $4+$ ). Alopecia is rarely seen with tamoxifen alone, but has been reported in occasional cases with vorinostat. While a significant number of patients had to be dose adjusted, another subset tolerated long-term administration of the drug combination for 2 years or longer.

\section{Anti-tumour efficacy of vorinostat and tamoxifen}

Responses were assessed by RECIST criteria version 1.0 (Therasse et al, 2000). Patients with bone-only disease were required to have
Table I Patient demographics, tumour characteristics, and treatment history

\begin{tabular}{|c|c|}
\hline Characteristic & \\
\hline $\begin{array}{l}\text { Number of patients } \\
\text { Measurable disease, } n(\%)\end{array}$ & $\begin{array}{c}43 \\
43(100 \%)\end{array}$ \\
\hline $\begin{array}{l}\text { Age, median (range), years } \\
\quad<65 \\
>65\end{array}$ & $\begin{array}{l}56(33-7 \mid) \\
37(86 \%) \\
6(14 \%)\end{array}$ \\
\hline $\begin{array}{l}\text { Race and ethnicity, n (\%) } \\
\text { Caucasian } \\
\text { Asian } \\
\text { African American } \\
\text { Non Hispanic } \\
\text { Hispanic }\end{array}$ & $\begin{aligned} 36 & (84 \%) \\
5 & (12 \%) \\
2 & (5 \%) \\
39 & (91 \%) \\
4 & (9 \%)\end{aligned}$ \\
\hline $\begin{array}{l}\text { Performance status, median (range) } \\
\text { Body mass index, } \mathrm{kg} \mathrm{m}^{-2} \text {, median (range) }\end{array}$ & $\begin{aligned} & \text { ( }(0-2) \\
27.9 & (20.7-44.2)\end{aligned}$ \\
\hline $\begin{array}{l}\text { Visceral disease, } n \text { (\%) } \\
\text { Yes } \\
\text { No }\end{array}$ & $\begin{array}{l}32(74 \%) \\
11(26 \%)\end{array}$ \\
\hline $\begin{array}{l}\text { Hormone receptor status, } n \text { (\%) } \\
\text { ER+/PR+ } \\
\text { ER+/PR- } \\
\text { ER+/PR unknown } \\
\text { ER-/PR+ }\end{array}$ & $\begin{array}{l}25(58 \%) \\
17(40 \%) \\
1(2 \%) \\
0(0 \%)\end{array}$ \\
\hline $\begin{array}{l}\text { HER amplification, } n \text { (\%) } \\
\text { Amplified } \\
\text { Non-amplified }\end{array}$ & $\begin{array}{r}5(12 \%) \\
38(88 \%)\end{array}$ \\
\hline $\begin{array}{l}\text { Prior endocrine therapy for metastatic disease, } n \text { ( } \\
0 \text { prior endocrine } \\
\text { I prior endocrine therapy } \\
2 \text { prior endocrine therapies }\end{array}$ & $\begin{array}{c}3(7 \%) \\
25(58 \%) \\
15(35 \%)\end{array}$ \\
\hline $\begin{array}{l}\text { Adjuvant endocrine therapy, } n \text { (\%) } \\
\text { Tamoxifen } \\
\text { Aromatase inhibitor }\end{array}$ & $\begin{array}{l}34(79 \%) \\
25(58 \%) \\
16(37 \%)\end{array}$ \\
\hline Aromatase inhibitor for metastatic disease & $35(81 \%)$ \\
\hline $\begin{array}{l}\text { Prior chemotherapy for metastatic disease, } n(\%) \\
\text { Regimens: median (range) }\end{array}$ & $\begin{array}{r}28(65 \%) \\
1(0-3)\end{array}$ \\
\hline Cycles delivered, median (range) & $4(1-27+)$ \\
\hline
\end{tabular}

Abbreviations: $\mathrm{ER}=$ oestrogen receptor; $\mathrm{PR}=$ progesterone receptor; $\mathrm{HER}=$ human epidermal growth factor receptor.

at least one lesion that measured $1 \mathrm{~cm}$ and was measurable by CT or magnetic resonance imaging. The study proceeded through the second stage after confirmed objective responses were seen in 4 of the first 18 patients, and grade 3 and 4 toxicities in $<4$ patients. Confirmed objective responses by RECIST criteria were seen in 8 out of $43(19 \%)$ patients and stable disease for $\geqslant 24$ weeks in 9 out of $43(21 \%)$ patients. Two of the patients with stable disease showed a complete metabolic response with disappearance of FGD-PET avidity after an initially increased metabolic activity by fluoro-deoxy glucose (FDG) positron emission tomogram (PET). The median number of cycles delivered was four. The time to progression was 10.3 months $(6-30+$ months) with an overall median survival of 29 months (95\% confidence interval (95\% CI): $20-38.5$ months). At the time of this report, two patients remain on study treatment.

All of the patients with a response or clinical benefit had progressed on at least one prior aromatase inhibitor, and 8 out of $17(47 \%)$ patients had received prior adjuvant tamoxifen. 
Responses were seen in patients with or without visceral disease, and responses were maintained in patients despite the need for dose adjustments in 8 out of 17 patients. Hormone receptor expression and prior treatment history of the responders are depicted in Table 3.

\section{Pharmacodynamics}

Previous studies with vorinostat and valproic acid in patients with solid tumour malignancies suggested that the induction of histone $\mathrm{H} 4$ acetylation in tumour cells was comparable to the increase in histone H4 acetylation seen in PBMCs (Munster et al, 2007, 2009a). Similarly, the drug-induced changes in histone acetylation in tumour and PBMCs mimicked those seen in cultured breast cancer cells treated with vorinostat at comparable doses. Prior studies further suggested that the assessment of histone acetylation

Table 2 Common Terminology Criteria of Adverse Events, grades $(2-4)$, by number of patients (\%)

\begin{tabular}{lllc}
\hline & Grade 2 & Grade 3 & Grade 4 \\
\hline Non-haematological toxicities (N=43) & & & \\
$\quad$ Fatigue & $8(19 \%)$ & $7(16 \%)$ & \\
Nausea & $8(19 \%)$ & $2(5 \%)$ & \\
Vomiting & $2(5 \%)$ & $2(5 \%)$ & \\
Diarrhoea & $7(16 \%)$ & $1(2 \%)$ & $3(7 \%)$ \\
DVT/PE & $7(16 \%)$ & $4(9 \%)$ & - \\
Anorexia/weight loss & $2(5 \%)$ & $1(2 \%)$ & - \\
Mucositis & $3(7 \%)$ & $1(2 \%)$ & - \\
Hepatic dysfunction (ALT/AST) & $5(12 \%)$ & $1(2 \%)$ & - \\
Hyperglycaemia & $4(9 \%)$ & - & - \\
Alopecia & & & \\
& $4(9 \%)$ & $3(7 \%)$ & $1(2 \%)$ \\
Haematological toxicities (N=43) & $4(9 \%)$ & $1(2 \%)$ & - \\
Thrombocytopenia & $7(12 \%)$ & $7(16 \%)$ & - \\
Leukopenia & $5(12 \%)$ & $6(14 \%)$ & - \\
Neutropenia \\
Lymphopenia \\
Anaemia
\end{tabular}

Abbreviations: $A L T=$ alanine aminotransferase; $A S T=$ aspartate aminotransferase; $\mathrm{DVT}=$ deep-vein thrombosis; $\mathrm{PE}=$ pulmonary embolism required much less tissue or fewer cells when using immunofluorescence and was feasible in tumour tissues obtained by fine needle administration. Prior studies suggested that histone $\mathrm{H} 4$ acetylation was more robust than histone $\mathrm{H} 3$ acetylation (Munster et al, 2007, 2009a). Hence for this study, changes in histone H4 acetylation in PBMCs were measured by immunofluorescence first and repeated by western blot if sufficient material was available. Complete pre- and day 8 post-treatment samples were available in 36 out of 43 patients (Figures 1 and 2). Missing data sets were in part due to the insufficient number of PBMCs as a result of myelosuppression in a subset of the patients, which happened early in the course of treatment. The mean increase in histone $\mathrm{H} 4$ acetylation was $20 \%$ over baseline and normalised for panhistone $\mathrm{H} 3$ expression (CI: 14-26\%) for the entire group. In patients with a partial response or stable disease for $>24$ weeks, the treatment-induced mean change in $\mathrm{H} 4$ histone acetylation was $39 \%$ (CI: $26-51 \%$ ) compared with $10 \%$. In our experience, the changes in histone acetylation by immunofluorescence were typically less pronounced than those seen by western blot analysis. To confirm the correlation of increased histone acetylation in responding $v s$ non-responding patients, pre- and post-treatment samples were assessed for histone acetylation changes by western blot analysis. Consistent with the immunofluorescence analysis, statistically more pronounced histone $\mathrm{H} 4$ acetylation was found in patients with a response or stable disease (430\% increase (CI: 219-633)) vs non-responders (6\% (CI: -7-21), $P=0.042$ ). As seen with our prior studies, the numerical changes were more robust, the number of patients with no change in histone acetylation were almost identical. However, immunofluorescence requires much less tissue and allows the assessment of histone acetylation in tumours cells obtained by fine needle aspiration (data not shown).

While several reports suggest a number of HDAC inhibitor targets, both histone and non-histone, as relevant for their observed anti-tumour activity, our preclinical data suggest that the depletion of HDAC2 by siRNA is sufficient, as its depletion mimics the effects of an HDAC inhibitor-tamoxifen combination in vitro. Thus, we believe HDAC2 represents the relevant target for achieving synergy with tamoxifen. We have further shown that the select depletion of HDAC2 may be important in the modification of ER signalling (Bicaku et al, 2008). Similarly, baseline expression

Table 3 Tumour characteristics, prior treatment history, and dose modifications in patients with a response or clinical benefit

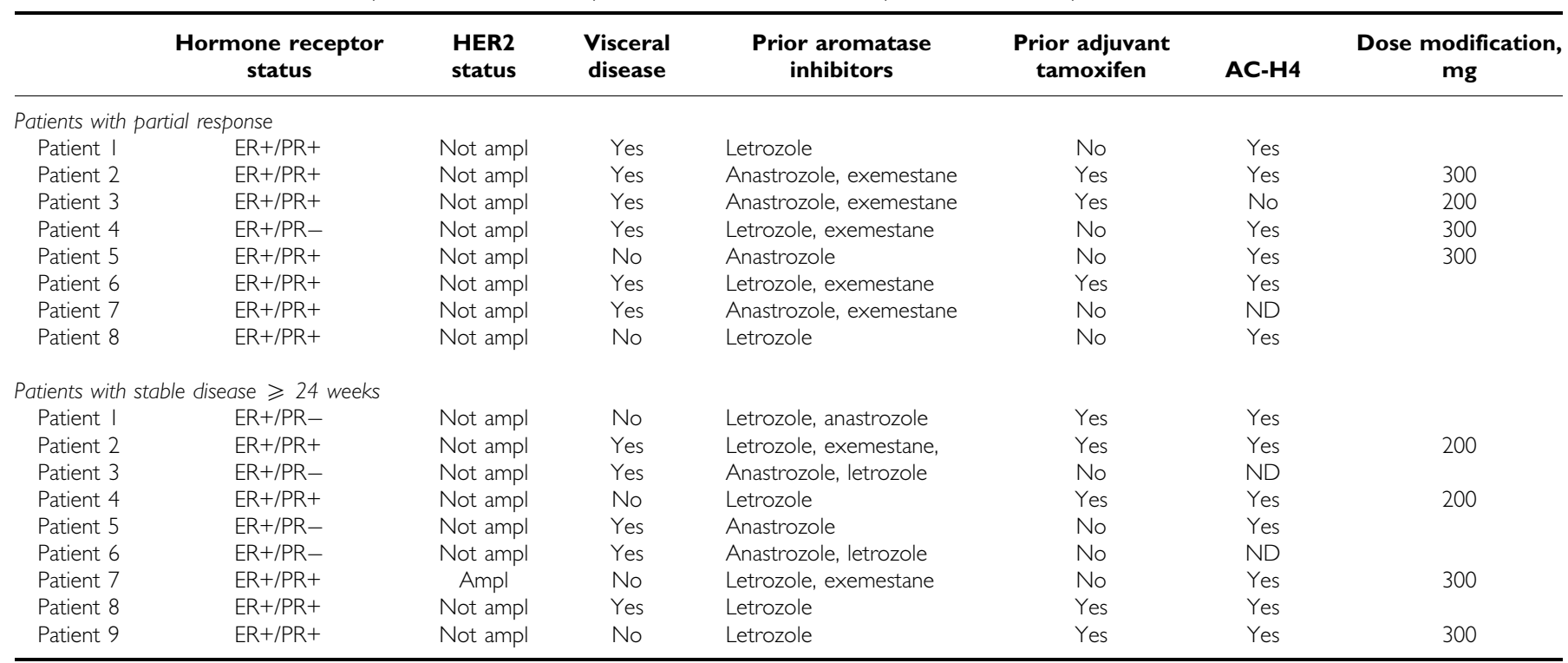

Abbreviations: $\mathrm{Ampl}=$ amplified; $\mathrm{HER} 2=$ human epidermal growth factor receptor 2; $\mathrm{AC}-\mathrm{H} 4=$ change in acetyl-H4 expression; $\mathrm{ND}=\mathrm{no}$ data; $\mathrm{ER}=$ oestrogen receptor; $\mathrm{PR}=$ progesterone receptor. 


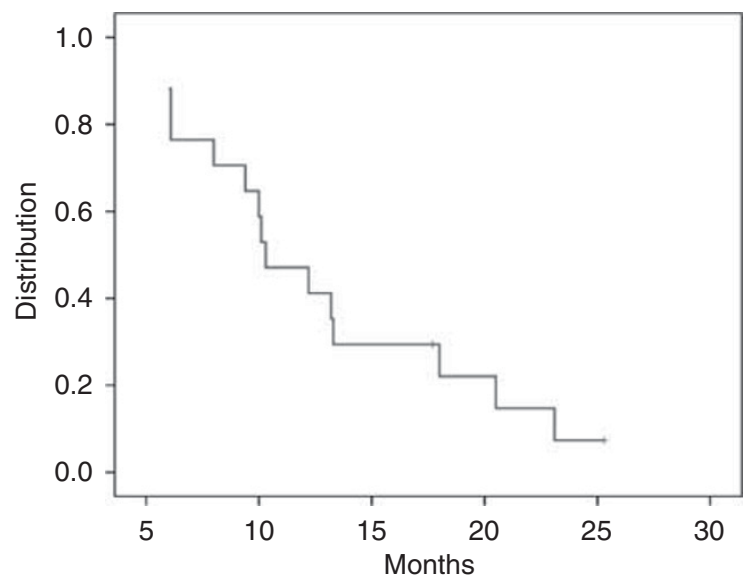

Duration of response

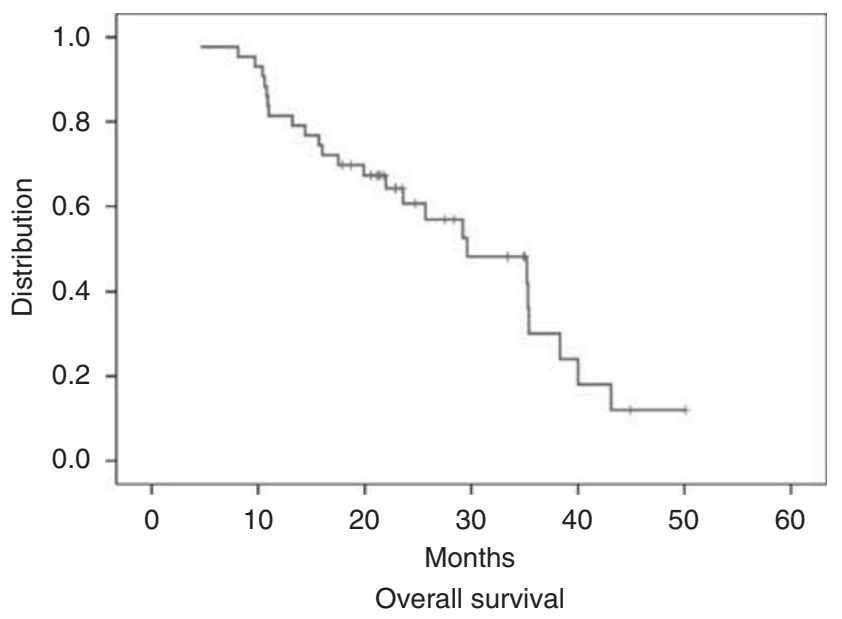

Figure I Median duration of response and overall survival.

of HDAC2 was higher in responding $v s$ non-responding patients. Measured in relative expression to pan-histone H3 expression, baseline HDAC2 expression in responders (R) was 2.48 times compared with 1.88 times in non-responders (NR) $(P=0.040$, Figure 2B).

In previous studies, we have shown that the change in histone acetylation correlates with baseline expression of HDAC2 (Munster et al, 2007, 2009a) in PBMC and tumour cells. In this study, higher baseline expression of HDAC2 in PBMCs was associated with a more pronounced increase in histone $\mathrm{H} 4$ acetylation (Figure $3 \mathrm{~A}$ ) $(P=0.003$, Pearson's correlation coefficient $=0.519)$.

Dose adjustments were required in $20(47 \%)$ of 43 treated patients. To determine whether the change in acetylation was predictive of toxicity, acetylation in patients with grade 3 and 4 toxicities $(n=20)$ was plotted against acetylation in those with grade 1 and 2 toxicities $(n=23)$ only. Changes in acetyl-H4 were not associated with severity of haematological or non-haematological toxicity $(P=0.49$, Figure $3 \mathrm{~B})$.

\section{DISCUSSION}

This is the first clinical study to evaluate the benefits of combining an HDAC inhibitor with an anti-oestrogen in patients with advanced ER-positive breast cancer. The rationale for this combination stems from extensive preclinical data suggesting epigenetic modulation and post-translational modification of the
A

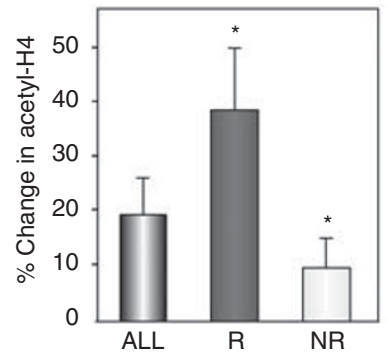

B
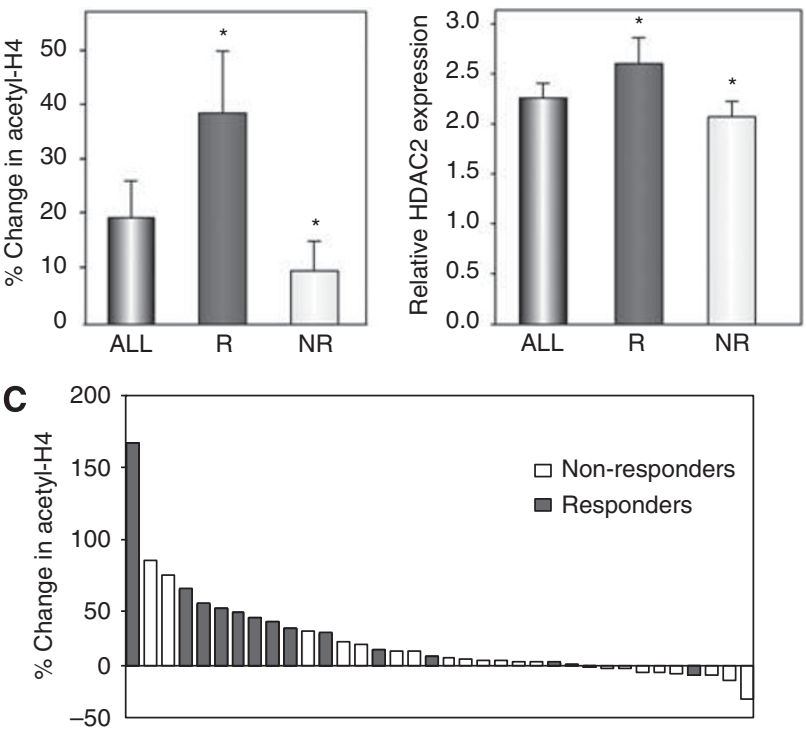

Figure 2 Percent change in acetyl-H4 (day 8 post-treatment pre-dose) in PBMCs by immunofluorescence normalised to pan-histone $\mathrm{H} 3$ expression. (A) Mean change of acetyl-H4 and (B) mean baseline HDAC2 expression relative to pan-histone $\mathrm{H} 3$ expression in all patients, in responders ( $R$; response or clinical benefit $\geqslant 24$ weeks) and in nonresponders (NR). *Statistical significance for acetyl-H4, $P=0.022$ ( $R$ vs NR) and HDAC2, $P=0.04$ (R vs NR). (C) Waterfall plot of acetyl-H4 in (ם) responders and $(\square)$ non-responders $(n=36)$.
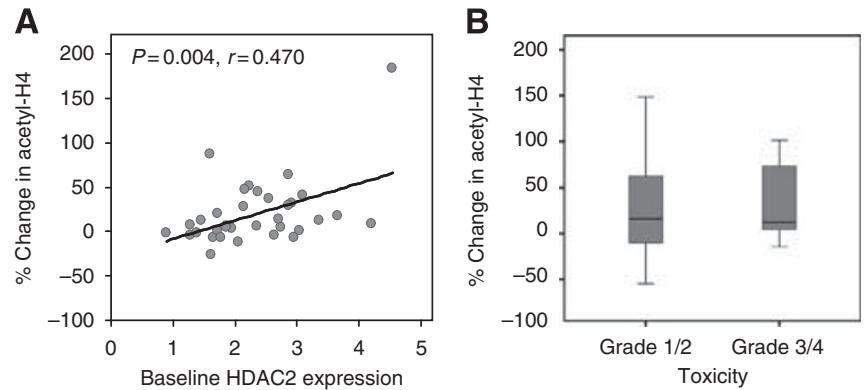

Figure 3 (A) Scatter plot of baseline histone deacetylase 2 (HDAC2) expression vs percent change in acetyl-H4 in PBMCs (day 8 post-treatment pre-dose) by immunofluorescence normalised to pan-histone $\mathrm{H} 3$ expression, analysed by Pearson's correlation $(P=0.004$, correlation coefficient of 0.470 ). (B) Percent change in acetyl- $\mathrm{H} 4$ in patients with less severe (grade $1 / 2$ ) and more severe (grade $3 / 4$ ) haematological or non-haematological toxicities $(P=0.49)$.

ER by HDAC inhibitors enhances the anti-tumour effects of tamoxifen (Yang et al, 2001; Kawai et al, 2003; Alao et al, 2004; Jang et al, 2004; Saji et al, 2005; Kawai and Arinze, 2006; Sharma et al, 2006; Fiskus et al, 2007; Zhou et al, 2007; Bicaku et al, 2008). We found that treatment of cultured breast cancer cells with vorinostat leads to downregulation and reversal of tamoxifeninduced stabilisation of the ER (Bicaku et al, 2008). The antitumour activity of tamoxifen is primarily anti-proliferative. In the presence of an HDAC inhibitor, however, we find that tamoxifen induces apoptosis rather than growth arrest. Further studies suggest that this interaction is mediated through inhibition of HDAC2 (Munster et al, 2007, 2009b; Bicaku et al, 2008; Marchion et al, 2009). Select depletion of HDAC2 by small interfering RNAs mimics the effects of an HDAC inhibitor on the ER and its 
downstream signalling (Bicaku et al, 2008). This led us to pursue a clinical trial evaluating the addition of an HDAC inhibitor to tamoxifen for the treatment of women with ER-positive breast cancer who had progressed on prior hormonal therapy.

Vorinostat was the first HDAC inhibitor approved by the FDA for the treatment of cancer, specifically cutaneous T-cell lymphoma. In addition to the effects in patients with cutaneous T-cell lymphoma, HDAC inhibitors appear to be active in Hodgkin's lymphoma and other haematological malignancies (Duvic et al, 2007). In contrast, the anti-tumour effects of vorinostat in solid tumour malignancies have been less evident. Vorinostat has been tested as a single agent in patients with metastatic breast cancer. Although disease stabilisation was observed in $30 \%$ of the patients, no clinical responses were achieved (Luu et al, 2008). Similarly, despite reported efficacy as single agents in several preclinical models, clinical benefit with HDAC inhibitors in several other solid tumour malignancies has been modest, limited mostly to disease stabilisation. Preclinical data from our laboratory and others suggest that HDAC inhibitors have the ability to re-sensitise tamoxifen-resistant cells to hormone therapy, and has been hypothesised to prevent the emergence of hormone therapy resistance (Yang et al, 2001; Zhou et al, 2007; Thomas et al, 2011).

This study's findings suggest that the addition of the HDAC inhibitor vorinostat to tamoxifen results in durable responses ( 8 out of $43,19 \%$ ) and prolonged disease stabilisation ( 9 out of 43 , $21 \%)$ in patients who had progressed on at least one prior aromatase inhibitor. Further, more than half of the patients had previously progressed on adjuvant tamoxifen. A significant proportion of the patients also received chemotherapy. The expected response rate in this patient population, based on the EFECT trial, is $7.6 \%$ for fulvestrant and $6.7 \%$ for exemestane (Chia et al, 2008). Two more contemporary trials suggested that while stable disease is achievable, objective, and durable responses are rare. The first trial reported disease stabilisation, but no objective responses using estradiol in this setting (Ellis et al, 2009). The second trial comparing tamoxifen plus placebo $v s$ tamoxifen plus EGFR inhibitor gefitinib demonstrated that tamoxifen and placebo treatment resulted in a $15 \%$ objective response rate in patients with newly diagnosed metastatic breast cancer or recurrent disease after adjuvant tamoxifen. However, in the stratum best comparable to the patient population in this study, patients recurring on or not responding to prior aromatase therapy, no objective responses were observed (Osborne et al, 2011). While in this study, the observed anti-tumour activity of $19 \%$ confirmed partial responses by RECIST criteria in this heavily pre-treated patient population is therefore very encouraging. Table 3 shows that responses were seen in patients after progression on two or three prior endocrine therapies and chemotherapy.

Furthermore, the correlative studies accompanying this clinical trial suggest that enrichment of responsive patients may be feasible. The pharmacological effects of vorinostat were evaluated in PBMCs. A statistically significant increase in histone $\mathrm{H} 4$ acetylation compared with baseline was observed in only 21 out of $36(58 \%)$ patients (Figure 2). This suggests that 15 out of 36 patients $(42 \%$ of the evaluated patients) did not reach vorinostat plasma levels that were high enough to induce a change in histone acetylation, or did not express the appropriate histone target. Several studies with vorinostat have shown that histone acetylation occurs at lower concentrations than those required for the modulation of other targets. Our data suggest that day 8 histone acetylation is a strong predictor of response. We have shown previously that vorinostat-induced histone acetylation in PBMCs is comparable to histone acetylation in tumour cells (Munster et al, 2009a). The mean increase in histone $\mathrm{H} 4$ acetylation for all patients in this study was 20\% (95\% CI: $14-26 \%$ ) over baseline when treated with $400 \mathrm{mg}$ of vorinostat. This may appear lower than has been previously reported when histone acetylation was measured by western blot analysis (Kelly et al, 2003). The effects, however, were comparable to our findings from other studies where histone acetylation was measured by immunofluorescence. We found that the observed changes in histone acetylation induced by $400 \mathrm{mg}$ oral vorinostat given daily in this study were comparable to the results observed in a dose escalation phase I trial evaluating vorinostat in combination with the anthracycline doxorubicin, conducted by our group (Munster et al, 2009a). We reported a $25 \%$ increase in mean histone acetylation (95\% CI: 3-47) on day 3 in patients receiving $400 \mathrm{mg}$ of vorinostat, with mean plasma levels of $114 \mathrm{~nm}$ (95\% CI: $37-191)$. Patients treated with higher doses of the HDAC inhibitor resulted in a $20 \%$ increase in median $\mathrm{H} 4$ histone acetylation (95\% CI: $29-70)$ at $600 \mathrm{mg}$ of vorinostat, and $84 \%(95 \%$ CI: 54-114) for $800 \mathrm{mg}$. Corresponding mean vorinostat levels were $256 \mathrm{~nm}$ (95\% CI: $133-378)$ and $760 \mathrm{~nm}$ (95\% CI: $598-924)$, respectively (Munster et al, 2009a).

To confirm that certain patients did not show a change in acetylation, we measured histone modification changes alternatively by western blot analysis. Although the range of histone acetylation measured by western blot analysis was numerically greater, a significant change in acetyl-H4 was only observed in $57 \%$ of the patients, comparable to the findings of the immunofluorescence analysis.

Furthermore, as shown in previous studies, baseline expression of HDAC2 is positively correlated with a change in histone $\mathrm{H} 4$ acetylation (Figure 3A), suggesting $\mathrm{HDAC} 2$ is a potential biomarker and important pharmacological target of vorinostat.

Responders were also more likely to exhibit elevated histone acetylation following treatment and increased baseline HDAC2 expression (Table 3; Figures 2A and B). An increase in histone acetylation was observed in 13 of 14 evaluable responders (Figure 2C). Thus, by identifying hyperacetylators at the initiation of treatment, patients most likely to benefit from this treatment would be enriched. Furthermore, determining initial acetylation response may provide direction for vorinostat dose modification.

Vorinostat and tamoxifen treatment was well tolerated by many of the patients and long-term exposure of up to 2 years was feasible. A subgroup of patients required dose modifications, however, due to grade 3 and 4 toxicities. In addition to myelosuppression, the predominant toxicities were fatigue and anorexia. These toxicities have been well described for vorinostat, and are less likely due to an interaction between vorinostat and tamoxifen. In the fourth week of each cycle (tamoxifen alone treatment), patients reported temporary alleviation from nausea, fatigue, anorexia, and myelosuppression. Notably, dose modification did not appear to compromise response, as 8 of the 17 patients with clinical benefit were dose adjusted, and maintained their response. Observed toxicities did not correlate with changes in histone acetylation (Figure 3), suggesting they may be the result of off target effects.

In support of our preclinical findings, baseline HDAC2 expression in patients' PBMCs correlated with the degree of histone acetylation. Thus, the assessment of baseline HDAC2 expression may predict a patient's molecular response. Patients with low HDAC2 expression, or those who do not show a change in acetylation, could then be removed from study or receive higher doses of the HDAC inhibitor if feasible.

In summary, this trial suggests that the addition of vorinostat to tamoxifen in patients with hormone receptor-positive breast cancer results in tumour regression or prolonged disease stabilisation in $40 \%$ of the patients who had progressed on prior hormonal therapy and chemotherapy. Although the current study may be limited by its sample size, the results are greater than those of contemporary studies testing endocrine therapy in second- and third-line therapy with response rates reported in $<10 \%$ of the patients, or no responses when measured by RECIST criteria (Chia et al, 2008). However, a randomised trial is required to determine the effects of the combination over the potential efficacy of tamoxifen alone. Pharmacodynamic assessment of 
vorinostat-induced histone $\mathrm{H} 4$ acetylation and HDAC2 expression at baseline was strong predictors of biological activity and clinical benefit. This suggests that the absence of histone acetylation could be used as an early negative predictor for patients who are not likely to benefit. These patients could then be removed from study, or be treated with a higher dose. The observed anti-tumour efficacy warrants further testing of HDAC2 inhibitors and hormonal therapy, yet the development of rapid acetylation bioassays and assessment of baseline HDAC2 expression in tumours in future studies may provide a feasible method to enrich for patients more likely to benefit.

\section{REFERENCES}

Alao JP, Lam EW, Ali S, Buluwela L, Bordogna W, Lockey P, Varshochi R, Stavropoulou AV, Coombes RC, Vigushin DM (2004) Histone deacetylase inhibitor trichostatin A represses estrogen receptor alpha-dependent transcription and promotes proteasomal degradation of cyclin D1 in human breast carcinoma cell lines. Clin Cancer Res 10: 8094-8104

Bicaku E, Marchion DC, Schmitt M, Munster PN (2008) Selective inhibition of histone deacetylase 2 silences progesterone receptor mediated signaling. Cancer Res 68: 1513-1519

Bonneterre J, Buzdar A, Nabholtz JM, Robertson JF, Thurlimann B, von Euler M, Sahmoud T, Webster A, Steinberg M (2001) Anastrozole is superior to tamoxifen as first-line therapy in hormone receptor positive advanced breast carcinoma. Cancer 92: 2247-2258

Chen TT, Ng TH (1998) Optimal flexible designs in phase II clinical trials. Stat Med 17: $2301-2312$

Chia S, Gradishar W, Mauriac L, Bines J, Amant F, Federico M, Fein L, Romieu G, Buzdar A, Robertson JF, Brufsky A, Possinger K, Rennie P, Sapunar F, Lowe E, Piccart M (2008) Double-blind, randomized placebo controlled trial of fulvestrant compared with exemestane after prior nonsteroidal aromatase inhibitor therapy in postmenopausal women with hormone receptor-positive, advanced breast cancer: results from EFECT. J Clin Oncol 26: $1664-1670$

Duvic M, Talpur R, Ni X, Zhang C, Hazarika P, Kelly C, Chiao JH, Reilly JF, Ricker JL, Richon VN, Frankel S (2007) Phase II trial of oral vorinostat (suberoylanilide hydroxamic acid, SAHA) for refractory cutaneous T-cell lymphoma (CTCL). Blood 109: 31-39

Early Breast Cancer Trialists' Collaborative Group (EBCTCG) (2005) Effects of chemotherapy and hormonal therapy for early breast cancer on recurrence and 15-year survival: an overview of the randomised trials. Lancet 365: 1687-1717

Ellis MJ, Gao F, Dehdashti F, Jeffe DB, Marcom PK, Carey LA, Dickler MN, Silverman P, Fleming GF, Kommareddy A, Jamalabadi-Majidi S, Crowder R, Siegel BA (2009) Lower-dose vs high-dose oral estradiol therapy of hormone receptor-positive, aromatase inhibitor-resistant advanced breast cancer: a phase 2 randomized study. JAMA 302: 774-780

Fiskus W, Ren Y, Mohapatra A, Bali P, Mandawat A, Rao R, Herger B, Yang Y, Atadja P, Wu J, Bhalla K (2007) Hydroxamic acid analogue histone deacetylase inhibitors attenuate estrogen receptor-alpha levels and transcriptional activity: a result of hyperacetylation and inhibition of chaperone function of heat shock protein 90. Clin Cancer Res 13: $4882-4890$

Galanis E, Jaeckle KA, Maurer MJ, Reid JM, Ames MM, Hardwick JS, Reilly JF, Loboda A, Nebozhyn M, Fantin VR, Richon VM, Scheithauer B, Giannini C, Flynn PJ, Moore Jr DF, Zwiebel J, Buckner JC (2009) Phase II trial of vorinostat in recurrent glioblastoma multiforme: a north central cancer treatment group study. J Clin Oncol 27: 2052-2058

Hodges-Gallagher L, Valentine CD, Bader SE, Kushner PJ (2006) Inhibition of histone deacetylase enhances the anti-proliferative action of antiestrogens on breast cancer cells and blocks tamoxifen-induced proliferation of uterine cells. Breast Cancer Res Treat 105(3): 297-309

Jang ER, Lim SJ, Lee ES, Jeong G, Kim TY, Bang YJ, Lee JS (2004) The histone deacetylase inhibitor trichostatin A sensitizes estrogen receptor alpha-negative breast cancer cells to tamoxifen. Oncogene 23: 1724- 1736

Jemal A, Siegel R, Xu J, Ward E (2010) Cancer statistics, 2010. CA Cancer J Clin 60: 277-300

Kawai H, Li H, Avraham S, Jiang S, Avraham HK (2003) Overexpression of histone deacetylase HDAC1 modulates breast cancer progression by negative regulation of estrogen receptor alpha. Int J Cancer 107: 353-358

\section{ACKNOWLEDGEMENTS}

We thank all of the patients and their families for their willingness to participate, as well as the clinical staff members of the Moffitt Cancer Center and University of California, San Francisco, who assisted us in this trial. We also thank Rasa Hamilton at the Moffitt Cancer Center for editorial assistance. This work was supported in part by a research grant from the Investigator-Initiated Studies Program of Merck Sharp \& Dohme Corp. The opinions expressed in this paper are those of the authors and do not necessarily represent those of Merck Sharp \& Dohme Corp.

Kawai Y, Arinze IJ (2006) Valproic acid-induced gene expression through production of reactive oxygen species. Cancer Res 66: 6563-6569

Kelly WK, O'Connor OA, Krug LM, Chiao JH, Heaney M, Curley T, MacGregore-Cortelli B, Tong W, Secrist JP, Schwartz L, Richardson S, Chu E, Olgac S, Marks PA, Scher H, Richon VM (2005) Phase I study of an oral histone deacetylase inhibitor, suberoylanilide hydroxamic acid, in patients with advanced cancer. J Clin Oncol 23: 3923-3931

Kelly WK, Richon VM, O’Connor O, Curley T, MacGregor-Curtelli B, Tong W, Klang M, Schwartz L, Richardson S, Rosa E, Drobnjak M, CordonCordo C, Chiao JH, Rifkind R, Marks PA, Scher H (2003) Phase I clinical trial of histone deacetylase inhibitor: suberoylanilide hydroxamic acid administered intravenously. Clin Cancer Res 9: 3578-3588

Luu TH, Morgan RJ, Leong L, Lim D, McNamara M, Portnow J, Frankel P, Smith DD, Doroshow JH, Wong C, Aparicio A, Gandara DR, Somlo G (2008) A phase II trial of vorinostat (suberoylanilide hydroxamic acid) in metastatic breast cancer: a California Cancer Consortium study. Clin Cancer Res 14: 7138-7142

Marchion DC, Bicaku E, Turner JG, Schmitt ML, Morelli DR, Munster PN (2009) HDAC2 regulates chromatin plasticity and enhances DNA vulnerability. Mol Cancer Ther 8: 794-801

Mouridsen H, Gershanovich M, Sun Y, Perez-Carrion R, Boni C, Monnier A, Apffelstaedt J, Smith R, Sleeboom HP, Janicke F, Pluzanska A, Dank M, Becquart D, Bapsy PP, Salminen E, Snyder R, Lassus M, Verbeek JA, Staffler B, Chaudri-Ross HA, Dugan M (2001) Superior efficacy of letrozole versus tamoxifen as first-line therapy for postmenopausal women with advanced breast cancer: results of a phase III study of the International Letrozole Breast Cancer Group. J Clin Oncol 19: 2596-2606

Munster P, Marchion D, Bicaku E, Lacevic M, Kim J, Centeno B, Daud A, Neuger A, Minton S, Sullivan D (2009a) Clinical and biological effects of valproic acid as a histone deacetylase inhibitor on tumor and surrogate tissues: phase I/II trial of valproic acid and epirubicin/FEC. Clin Cancer Res 15: $2488-2496$

Munster P, Marchion D, Bicaku E, Schmitt M, Lee JH, DeConti R, Simon G, Fishman M, Minton S, Garrett C, Chiappori A, Lush R, Sullivan D, Daud A (2007) Phase I trial of histone deacetylase inhibition by valproic acid followed by the topoisomerase II inhibitor epirubicin in advanced solid tumors: a clinical and translational study. J Clin Oncol 25: 1979-1985

Munster PN, Marchion D, Thomas S, Egorin M, Minton S, Springett G, Lee JH, Simon G, Chiappori A, Sullivan D, Daud A (2009b) Phase I trial of vorinostat and doxorubicin in solid tumours: histone deacetylase 2 expression as a predictive marker. Br J Cancer 101: 1044-1050

Nabholtz JM, Buzdar A, Pollak M, Harwin W, Burton G, Mangalik A, Steinberg M, Webster A, von Euler M (2000) Anastrozole is superior to tamoxifen as first-line therapy for advanced breast cancer in postmenopausal women: results of a North American multicenter randomized trial. Arimidex Study Group. J Clin Oncol 18: 3758-3767

O'Connor OA, Heaney ML, Schwartz L, Richardson S, Willim R, MacGregor-Cortelli B, Curly T, Moskowitz C, Portlock C, Horwitz S, Zelenetz AD, Frankel S, Richon V, Marks P, Kelly WK (2006) Clinical experience with intravenous and oral formulations of the novel histone deacetylase inhibitor suberoylanilide hydroxamic acid in patients with advanced hematologic malignancies. J Clin Oncol 24: 166-173

Osborne CK, Neven P, Dirix LY, Mackey JR, Robert J, Underhill C, Schiff R, Gutierrez C, Migliaccio I, Anagnostou VK, Rimm DL, Magill P, Sellers MV (2011) Gefitinib or placebo in combination with tamoxifen in patients with hormone receptor-positive metastatic breast cancer: a randomized phase II study. Clin Cancer Res 17(5): 1147-1159 
Saji S, Kawakami M, Hayashi S, Yoshida N, Hirose M, Horiguchi S, Itoh A, Funata N, Schreiber SL, Yoshida M, Toi M (2005) Significance of HDAC6 regulation via estrogen signaling for cell motility and prognosis in estrogen receptor-positive breast cancer. Oncogene 24: $4531-4539$

Sharma D, Saxena NK, Davidson NE, Vertino PM (2006) Restoration of tamoxifen sensitivity in estrogen receptor-negative breast cancer cells: tamoxifen-bound reactivated ER recruits distinctive corepressor complexes. Cancer Res 66: 6370-6378

Therasse P, Arbuck SG, Eisenhauer EA, Wanders J, Kaplan RS, Rubinstein L, Verweij J, Van Glabbeke M, van Oosterom AT, Christian MC, Gwyther SG (2000) New guidelines to evaluate the response to treatment in solid tumors. European Organization for Research and Treatment of Cancer, National Cancer Institute of the United States, National Cancer Institute of Canada. J Natl Cancer Inst 92: 205-216
Thomas S, Thurn KT, Bicaku E, Marchion DC, Munster PN (2011) Addition of a histone deacetylase inhibitor redirects tamoxifen-treated breast cancer cells into apoptosis, which is opposed by the induction of autophagy. Breast Cancer Res Treat; e-pub ahead of print 5 February 2011

Yang X, Phillips DL, Ferguson AT, Nelson WG, Herman JG, Davidson NE (2001) Synergistic activation of functional estrogen receptor (ER)-alpha by DNA methyltransferase and histone deacetylase inhibition in human ER-alpha-negative breast cancer cells. Cancer Res 61: 7025-7029

Yi X, Wei W, Wang SY, Du ZY, Xu YJ, Yu XD (2008) Histone deacetylase inhibitor SAHA induces ERalpha degradation in breast cancer MCF-7 cells by CHIP-mediated ubiquitin pathway and inhibits survival signaling. Biochem Pharmacol 75: 1697-1705

Zhou Q, Atadja P, Davidson NE (2007) Histone deacetylase inhibitor LBH589 reactivates silenced estrogen receptor alpha (ER) gene expression without loss of DNA hypermethylation. Cancer Biol Ther 6(1): 64-69 